\title{
ASYMPTOTIC LIMITS AND STABILIZATION FOR THE 1D NONLINEAR MINDLIN-TIMOSHENKO SYSTEM*
}

\author{
F. D. ARARUNA $\cdot$ P. BRAZ E SILVA $\cdot$ E. ZUAZUA
}

DOI:

Received: 10 December 2009 / Revised: 23 February 2010

(C)The Editorial Office of JSSC \& Springer-Verlag Berlin Heidelberg 2010

\begin{abstract}
This paper shows how the so called von Kármán model can be obtained as a singular limit of a modified Mindlin-Timoshenko system when the modulus of elasticity in shear $k$ tends to infinity, provided a regularizing term through a fourth order dispersive operator is added. Introducing damping mechanisms, the authors also show that the energy of solutions for this modified Mindlin-Timoshenko system decays exponentially, uniformly with respect to the parameter $k$. As $k \rightarrow \infty$, the authors obtain the damped von Kármán model with associated energy exponentially decaying to zero as well.
\end{abstract}

Key words Mindlin-Timoshenko system, singular limit, uniform stabilization, vibrating beams, von Kármán system.

\section{Introduction}

The Mindlin-Timoshenko system of equations is a widely used and fairly complete mathematical model for describing the transverse vibrations of beams. It is a more accurate model than the Euler-Bernoulli one, since it also takes into account transverse shear effects. The Mindlin-Timoshenko system is used, for example, to model aircraft wings (see, e.g., [1]).

For a beam of length $L>0$, this one-dimensional nonlinear system reads as

$$
\begin{cases}\frac{\rho h^{3}}{12} \phi_{t t}-\phi_{x x}+k\left(\phi+\psi_{x}\right)=0 & \text { in } Q \\ \rho h \psi_{t t}-k\left(\phi+\psi_{x}\right)_{x}-\left[\psi_{x}\left(\eta_{x}+\frac{1}{2} \psi_{x}^{2}\right)\right]_{x}=0 & \text { in } Q \\ \rho h \eta_{t t}-\left(\eta_{x}+\frac{1}{2} \psi_{x}^{2}\right)_{x}=0 & \text { in } Q\end{cases}
$$

F. D. ARARUNA

Departamento de Matemática Universidade Federal da Paraiba, 58051-900, João Pessoa, PB, Brasil.

Email: fagner@mat.ufpb.br; pablo@dmat.ufpe.br.

P. BRAZ E SILVA

Departamento de Matemática, Universidade Federal de Pernambuco, 50740-540, Recife, PE, Brasil.

Email: pablo@dmat.ufpe.br.

E. ZUAZUA

Ikerbasque Research Professor, Basque Center for Applied Mathematics (BCAM), Bizkaia Technology Park, Building 500,E-48160, Derio, Basque Country, Spain. Email: zuazua@bcamath.org.

* This paper is partially supported by INCTMat, FAPESQ-PB, CNPq (Brazil) under Grant Nos. 308150/2008-2 and 620108/2008-8, the MICINN (Spain) under Grant No. MTM2008-03541, the Advanced Grant FP7-246775 NUMERIWAVES of the ERC, and the Project PI2010-04 of the Basque Government, also dedicated to Prof. D. L. Russell on his 70th birthday. 
where $Q=(0, L) \times(0, T)$, the interval $(0, L)$ is the segment occupied by the beam, and $T$ is a given positive time. In system (1), subscripts mean partial derivatives. The unknowns $\phi=\phi(x, t), \psi=\psi(x, t)$, and $\eta=\eta(x, t)$ represent, respectively, the angle of rotation, the vertical displacement, and the longitudinal displacement at time $t$ of the cross section located $x$ units from the end-point $x=0$. The constant $h>0$ represents the thickness of the beam which, in this model, is considered to be small and uniform with respect to $x$. The constant $\rho$ is the mass density per unit volume of the beam and the parameter $k$ is the so called modulus of elasticity in shear. It is given by the expression $k=\widehat{k} E h / 2(1+\mu)$, where $\widehat{k}$ is a shear correction coefficient, $E$ is the Young's modulus and $\mu$ is the Poisson's ratio, $0<\mu<1 / 2$. For more details concerning the Mindlin-Timoshenko hypotheses and governing equations ${ }^{[2-3]}$.

There is a large literature on this model, addressing problems of existence, uniqueness and asymptotic behavior in time when some damping effects are considered, as well as some other important properties (see [2-4] and references therein).

When one assumes the linear filament of the beam to remain orthogonal to the deformed middle surface, the transverse shear effects are neglected, and one obtains the so called von Kármán system (see [3]):

$$
\begin{cases}\rho h \psi_{t t}-\frac{\rho h^{3}}{12} \psi_{x x t t}+\psi_{x x x x}-\left[\psi_{x}\left(\eta_{x}+\frac{1}{2} \psi_{x}^{2}\right)\right]_{x}=0 & \text { in } Q, \\ \rho h \eta_{t t}-\left(\eta_{x}+\frac{1}{2} \psi_{x}^{2}\right)_{x}=0 & \text { in } Q .\end{cases}
$$

Note that neglecting the shear effects of the beam is formally equivalent to considering the modulus $k$ tending to infinity in system (1), since $k$ is inversely proportional to the shear angle. There is also an extensive literature about system (2) (see [2-3,5-6] and references therein).

In fact, since the pioneering work of D. L. Russell and coworkers summarized in his celebrated 1978 paper in SIAM Review ${ }^{[7]}$, the control and stabilization of wave processes have undergone a significant progress. It would be impossible to summarize here the variety and the depth of the results developed after his influential work. More recently, the subject has been developed even further using the so-called Hilbert Uniqueness Method (HUM) developed by Lions and presented, in particular, in his SIAM Review article of $1988^{[8]}$. Using HUM one can transform observability inequalities on the adjoint system (that can be derived using multipliers, non-harmonic Fourier series techniques, microlocal analysis, Carleman inequalities, etc) into controllability ones by means of a flexible variational technique. This allows one to prove, for instance, the existence of controls with minimal norm in different functional frameworks. The same observability inequalities can then be used to build feedback controllers and to get a variety of new stabilization results. There is also an extensive literature in this subject including wave, beam, plate, $\mathrm{KdV}$, and Schrödinger equations, among other models considered. The present article is a further contribution in this context, focusing mainly on the behavior of the exponential decay rate under a relevant singular perturbation in elasticity theory.

More precisely, in the context of systems of elasticity, it is well-known that reduced models are often singular limits of more complete ones. The issue of the dependence of the decay rate or, more generally, the stabilization properties with respect to this singular parameter is also an interesting subject that has been object of intensive research. The present paper is devoted to addressing this issue in the context of nonlinear models for vibrating beams. Our interest is to analyze the asymptotic limit of the nonlinear Mindlin-Timoshenko system (1), when the shear modulus $k$ tends to infinity. This problem was mentioned in [3], where it was conjectured that the system (1) approaches, in some sense, the von Kármán system (2) as $k \rightarrow \infty$.

In order to achieve our results, we perturb system (1) by a term of the type $\psi_{x x x x} / k$. More 
precisely, instead of system (1) we consider the modified system:

$$
\begin{cases}\frac{\rho h^{3}}{12} \phi_{t t}-\phi_{x x}+k\left(\phi+\psi_{x}\right)=0 & \text { in } Q \\ \rho h \psi_{t t}-k\left(\phi+\psi_{x}\right)_{x}-\left[\psi_{x}\left(\eta_{x}+\frac{1}{2} \psi_{x}^{2}\right)\right]_{x}+\frac{1}{k} \psi_{x x x x}=0 & \text { in } Q \\ \rho h \eta_{t t}-\left(\eta_{x}+\frac{1}{2} \psi_{x}^{2}\right)_{x}=0 & \text { in } Q\end{cases}
$$

and show the following:

i) The system (3) converges to system (2) as the parameter $k \rightarrow \infty$.

ii) Adding appropriate damping terms, there is a uniform (with respect to $k$ ) rate of decay for the total energy of the solutions of (3) as $t \rightarrow \infty$.

As a consequence of this analysis, we obtain a decay rate for the total energy of the solutions of the von Kármán system (as $t \rightarrow \infty$ ) as a singular limit of the uniform (with respect to $k$ ) decay rate of the energy of the Mindlin-Timoshenko system. Note, however, that our results need the fourth order regularizing term in the component $\psi$ for compactness purposes. Reproducing the results of this paper without that regularizing term is an interesting open problem. These results are proved under suitable boundary and initial conditions that we shall make precise below.

The connections between these two systems and the asymptotic limit problem of passing to the limit as $k$ tends to infinity have been recently investigated in a number of different situations. For the linear case, that is, in the absence of the term $\left[\psi_{x}\left(\eta_{x}+\frac{1}{2} \psi_{x}^{2}\right)\right]_{x}$, and without the equation for $\eta$, it was proved in [3] (see also [9]) that the linear Mindlin-Timoshenko system

$$
\left\{\begin{array}{l}
\frac{\rho h^{3}}{12} \phi_{t t}-\phi_{x x}+k\left(\phi+\psi_{x}\right)=0, \\
\rho h \psi_{t t}-k\left(\phi+\psi_{x}\right)_{x}=0
\end{array}\right.
$$

approaches, as $k \rightarrow \infty$, the Kirchhoff equation

$$
\rho h \psi_{t t}-\frac{\rho h^{3}}{12} \psi_{x x t t}+\psi_{x x x x}=0,
$$

under various boundary conditions. Controllability properties of these linear systems have also been studied. More precisely, using Fourier series decompositions and filtering techniques, it was shown in [9] that the exact controllability of the Kirchhoff system may be obtained as the limit, when $k \rightarrow \infty$, of a partial controllability property for the Mindlin-Timoshenko system. Note however that these techiques, based in the Fourier analysis, cannot be applied in the present nonlinear context. In [4], the authors proved the existence of a compact global attractor for system (4), for the two dimensional case, with nonlinear feedback forces. They also showed the limit of this attractor, as $k \rightarrow \infty$, to be closely related to the attractor of the limit system (5). The problem of singular perturbations and stabilization related to the nonlinear von Kármán model has also been intensively investigated. We refer, for instance, to [10-13], where these issues are addressed under various boundary conditions.

In the present paper we consider system (3) under Dirichlet boundary conditions for $\phi$, clamped ends for $\psi$, and Neumann boundary conditions on $\eta$ :

$$
\begin{cases}\phi(0, \cdot)=\phi(L, \cdot)=0 & \text { on } \quad(0, T), \\ \psi(0, \cdot)=\psi(L, \cdot)=\psi_{x}(0, \cdot)=\psi_{x}(L, \cdot)=0 & \text { on } \quad(0, T), \\ \eta_{x}(0, \cdot)=\eta_{x}(L, \cdot)=0 & \text { on } \quad(0, T),\end{cases}
$$


and the initial data

$$
\left\{\begin{array}{l}
\{\phi(\cdot, 0), \psi(\cdot, 0), \eta(\cdot, 0)\}=\left\{\phi_{0}, \psi_{0}, \eta_{0}\right\} \quad \text { in } \quad(0, L) \\
\left\{\phi_{t}(\cdot, 0), \psi_{t}(\cdot, 0), \eta_{t}(\cdot, 0)\right\}=\left\{\phi_{1}, \psi_{1}, \eta_{1}\right\} \quad \text { in } \quad(0, L)
\end{array}\right.
$$

In Section 2, we analyze the well-posedness of the system (3), (6), (7) in the corresponding finite energy space. In Section 3, we rigorously study the limit behavior of the Mindlin-Timoshenko system as $k \rightarrow \infty$ towards the von Kármán system. More precisely, we prove that solutions $\{\phi, \psi, \eta\}$ of (3), (6), (7) converge to $\left\{-\psi_{x}, \psi, \eta\right\}$ as $k \rightarrow \infty$, where $\{\psi, \eta\}$ solves system (2) with the same boundary conditions for $\psi$ and $\eta$. In Section 4 , adding damping terms, we prove an uniform (in $k$ ) exponential decay property for the solutions of (3), (6), (7). Finally, in Section 5 , we briefly discuss some related issues and open problems.

\section{Global Well-Posedness}

In this section, for the sake of completeness, we study existence and uniqueness of solutions for system (3), (6), (7). First of all, we introduce the Hilbert space

$$
\mathcal{X}=H_{0}^{1}(0, L) \times L^{2}(0, L) \times H_{0}^{2}(0, L) \times L^{2}(0, L) \times V \times H,
$$

where $V=H^{1}(0, L) \cap H$ and $H=\left\{v \in L^{2}(0, L) ; \int_{0}^{L} v(x) d x=0\right\}$, equipped with the norm

$$
\begin{aligned}
\left\|\left\{u_{1}, u_{2}, v_{1}, v_{2}, w_{1}, w_{2}\right\}\right\|_{\mathcal{X}}^{2}= & \left|u_{1 x}\right|^{2}+\frac{\rho h^{3}}{12}\left|u_{2}\right|^{2}+k\left|u_{1}+v_{1 x}\right|^{2}+\frac{1}{k}\left|v_{1 x x}\right|^{2} \\
& +\rho h\left|v_{2}\right|^{2}+\left|w_{1 x}\right|^{2}+\rho h\left|w_{2}\right|^{2},
\end{aligned}
$$

where $|\cdot|$ denotes the norm in $L^{2}(0, L)$.

Theorem 1 If $\left(\phi_{0}, \phi_{1}, \psi_{0}, \psi_{1}, \eta_{0}, \eta_{1}\right) \in \mathcal{X}$, then problem (3), (6), (7) has a unique weak solution in the class

$$
\{\phi, \psi, \eta\} \in C^{0}\left([0, \infty) ; H_{0}^{1}(0, L) \times H_{0}^{2}(0, L) \times V\right) \cap C^{1}\left([0, \infty) ;\left[L^{2}(0, L)\right]^{2} \times H\right) .
$$

Moreover, the energy $E_{k}(t)$, given by

$$
\begin{aligned}
E_{k}(t)=\frac{1}{2} & \left(\frac{\rho h^{3}}{12}\left|\phi_{t}(t)\right|^{2}+\rho h\left|\psi_{t}(t)\right|^{2}+\rho h\left|\eta_{t}(t)\right|^{2}+\left|\phi_{x}(t)\right|^{2}\right. \\
& \left.+k\left|\phi(t)+\psi_{x}(t)\right|^{2}+\left|\eta_{x}(t)+\frac{1}{2}\left[\psi_{x}(t)\right]^{2}\right|^{2}+\frac{1}{k}\left|\psi_{x x}(t)\right|^{2}\right),
\end{aligned}
$$

satisfies

$$
E_{k}(t)=E_{k}(0), \quad \forall t \geq 0
$$

Remark 1 Note that the energy $E_{k}(t)$ defines a norm in $\mathcal{X}$ which is equivalent to $\|\cdot\|_{\mathcal{X}}$.

Proof of Theorem 1 The problem (3), (6), (7) can be written as

$$
\left\{\begin{array}{l}
U_{t}=\mathcal{A} U+F(U) \\
U(0)=U_{0}
\end{array}\right.
$$


where

$$
\begin{gathered}
\mathcal{A}=\left[\begin{array}{cccccc}
0 & 1 & 0 & 0 & 0 & 0 \\
\frac{12}{\rho h^{3}}\left(\frac{\partial^{2}}{\partial x^{2}}-k\right) & 0 & -\frac{12 k}{\rho h^{3}} \frac{\partial}{\partial x} & 0 & 0 & 0 \\
0 & 0 & 0 & 1 & 0 & 0 \\
\frac{k}{\rho h} \frac{\partial}{\partial x} & 0 & \frac{k}{\rho h} \frac{\partial^{2}}{\partial x^{2}}-\frac{1}{\rho h k} \frac{\partial^{4}}{\partial x^{4}} & 0 & 0 & 0 \\
0 & 0 & 0 & 0 & 0 & 1 \\
0 & 0 & 0 & \frac{1}{\rho h} \frac{\partial^{2}}{\partial x^{2}} & 0
\end{array}\right], \quad U=\left[\begin{array}{c}
\phi \\
\phi^{\prime} \\
\psi \\
\psi^{\prime} \\
\eta \\
\eta^{\prime}
\end{array}\right], \\
F(U)=\left[\begin{array}{c}
0 \\
0 \\
0 \\
\left.\psi_{x}\left(\eta_{x}+\frac{1}{2} \psi_{x}^{2}\right)\right]_{x} \\
0 \\
\frac{1}{2}\left(\psi_{x}^{2}\right)_{x}
\end{array}\right], \text { and } U_{0}=\left[\begin{array}{c}
\phi_{0} \\
\phi_{1} \\
\psi_{0} \\
\psi_{1} \\
\eta_{0} \\
\eta_{1}
\end{array}\right] .
\end{gathered}
$$

It is easy to see that the operator $\mathcal{A}: D(\mathcal{A}) \subset \mathcal{X} \rightarrow \mathcal{X}$ with domain

$$
\begin{aligned}
D(\mathcal{A})= & {\left[H^{2}(0, L) \cap H_{0}^{1}(0, L)\right] \times H_{0}^{1}(0, L) \times\left[H^{4}(0, L) \cap H_{0}^{2}(0, L)\right] \times H_{0}^{1}(0, L) } \\
& \times W \times H^{1}(0, L),
\end{aligned}
$$

where

$$
W=\left\{v \in H^{2}(0, L) ; v_{x}(0)=v_{x}(L)=0\right\}
$$

is the infinitesimal generator of a semigroup of operators in $\mathcal{X}$. Indeed,

$$
\begin{aligned}
& \langle-\mathcal{A} U, U\rangle_{\mathcal{X}}=-\int_{0}^{L} \phi_{x} \phi_{x}^{\prime} d x-\int_{0}^{L} \phi_{x x} \phi^{\prime} d x+k \int_{0}^{L}\left(\phi+\psi_{x}\right) \phi^{\prime} d x-k \int_{0}^{L}\left(\phi^{\prime}+\psi_{x}^{\prime}\right)\left(\phi+\psi_{x}\right) d x \\
& -\frac{1}{k} \int_{0}^{L} \psi_{x x} \psi_{x x}^{\prime} d x-k \int_{0}^{L}\left(\phi+\psi_{x}\right)_{x} \psi^{\prime} d x+\frac{1}{k} \int_{0}^{L} \psi_{x x x x} \psi^{\prime} d x-\int_{0}^{L} \eta_{x} \eta_{x}^{\prime} d x-\int_{0}^{L} \eta_{x x} \eta^{\prime} d x=0 .
\end{aligned}
$$

On the other hand, given $\Phi=\{\alpha, \beta, \gamma, \zeta, \theta, \lambda\}^{\mathrm{T}} \in \mathcal{X}$, the system

$$
(-\mathcal{A}+I) U=\Phi \quad \text { in } \quad(0, L)
$$

has a unique solution $U=\left\{\phi, \phi^{\prime}, \psi, \psi^{\prime}, \eta, \eta^{\prime}\right\}^{\mathrm{T}} \in D(\mathcal{A})$. In fact, the system (11) reduces to

$$
\left\{\begin{array}{l}
\phi-\phi^{\prime}=\alpha, \\
-\frac{12}{\rho h^{3}} \phi_{x x}+\frac{12 k}{\rho h^{3}} \phi+\frac{12 k}{\rho h^{3}} \psi_{x}+\phi^{\prime}=\beta, \\
\psi-\psi^{\prime}=\gamma, \\
\frac{1}{\rho h k} \psi_{x x x x}-\frac{k}{\rho h} \psi_{x x}-\frac{k}{\rho h} \phi_{x}+\psi^{\prime}=\zeta, \\
\eta-\eta^{\prime}=\theta, \\
-\frac{1}{\rho h} \eta_{x x}+\eta^{\prime}=\lambda .
\end{array}\right.
$$


Solving system (12) is equivalent to finding

$$
\{\phi, \psi, \eta\} \in\left[H^{2}(0, L) \cap H_{0}^{1}(0, L)\right] \times\left[H^{4}(0, L) \cap H_{0}^{2}(0, L)\right] \times W
$$

such that

$$
\left\{\begin{array}{l}
\int_{0}^{L} \phi_{x} \xi_{x} d x+k \int_{0}^{L}\left(\phi+\psi_{x}\right) \xi d x+\frac{\rho h^{3}}{12} \int_{0}^{L} \phi \xi d x=\frac{\rho h^{3}}{12} \int_{0}^{L}(\alpha+\beta) \xi d x, \forall \xi \in H_{0}^{1}(0, L), \\
\frac{1}{k} \int_{0}^{L} \psi_{x x} \sigma_{x x} d x+k \int_{0}^{L}\left(\phi+\psi_{x}\right) \sigma_{x} d x+\rho h \int_{0}^{L} \psi \sigma d x=\rho h \int_{0}^{L}(\gamma+\zeta) \sigma d x, \forall \sigma \in H_{0}^{2}(0, L),
\end{array}\right.
$$

and

$$
\left\{\begin{array}{l}
-\eta_{x x}+\rho h \eta=\rho h(\theta+\lambda) \quad \text { in } \quad(0, L), \\
\eta_{x}(0)=\eta_{x}(L)=0 .
\end{array}\right.
$$

Define the bilinear form $b:\left[H_{0}^{1}(0, L) \times H_{0}^{2}(0, L)\right]^{2} \rightarrow \boldsymbol{R}$ by

$$
\begin{aligned}
b(\{\phi, \psi\},\{\xi, \sigma\})= & \int_{0}^{L} \phi_{x} \xi_{x} d x+\frac{\rho h^{3}}{12} \int_{0}^{L} \phi \xi d x+\frac{1}{k} \int_{0}^{L} \psi_{x x} \sigma_{x x} d x \\
& +k \int_{0}^{L}\left(\phi+\psi_{x}\right)\left(\xi+\sigma_{x}\right) d x+\rho h \int_{0}^{L} \psi \sigma d x,
\end{aligned}
$$

and the linear one $l: H_{0}^{1}(0, L) \times H_{0}^{2}(0, L) \rightarrow \boldsymbol{R}$ by

$$
l(\{\xi, \sigma\})=\frac{\rho h^{3}}{12} \int_{0}^{L}(\alpha+\beta) \xi d x+\rho h \int_{0}^{L}(\gamma+\zeta) \sigma d x .
$$

It is easy to see that $b$ is continuous and coercive, and that $l$ is continuous. So, due to the Lax-Milgram's theorem, there exists a unique $\{\phi, \psi\} \in H_{0}^{1}(0, L) \times H_{0}^{2}(0, L)$ such that

$$
b(\{\phi, \psi\},\{\xi, \sigma\})=l(\{\xi, \sigma\}), \forall\{\xi, \sigma\} \in H_{0}^{1}(0, L) \times H_{0}^{2}(0, L) .
$$

Considering vectors of the form $\{\xi, 0\}$, with $\xi \in H_{0}^{1}(0, L)$, and $\{0, \sigma\}$, with $\sigma \in H_{0}^{2}(0, L)$, one concludes that the pair $\{\phi, \psi\}$ found above satisfies (13). Furthermore, from $(12)_{1}$ and $(12)_{3}$, one has $\left\{\phi^{\prime}, \psi^{\prime}\right\} \in H_{0}^{1}(0, L) \times H_{0}^{2}(0, L)$. Applying classical elliptic regularity, it follows from (13) that $\{\phi, \psi\} \in H^{2}(0, L) \times H^{4}(0, L)$. Classical elliptic theory applied for the operator $-\frac{d^{2}}{d x^{2}}+\delta I$, $\delta>0$, with Neumann conditions, assures that (14) possesses only one solution in the class $\eta \in W$. In this way, one obtains the existence of a unique solution $\left\{\phi, \phi^{\prime}, \psi, \psi^{\prime}, \eta, \eta^{\prime}\right\} \in D(\mathcal{A})$ for system (11).

To prove existence and uniqueness of local solutions for the nonlinear problem, it remains to show that $F(U)$ is locally Lipschitz continuous in $\mathcal{X}$. In fact, if $U=\left\{\phi, \phi^{\prime}, \psi, \psi^{\prime}, \eta, \eta^{\prime}\right\}^{\mathrm{T}}$, $\widetilde{U}=\left\{\widetilde{\phi}, \widetilde{\phi^{\prime}}, \widetilde{\psi}, \widetilde{\psi^{\prime}}, \widetilde{\eta}, \widetilde{\eta}^{\prime}\right\}^{\mathrm{T}}$ belong to $\mathcal{X}$, we have

$$
\|F(U)-F(\widetilde{U})\|_{\mathcal{X}}^{2}=\rho h\left(|f|^{2}+|g|^{2}\right)
$$

where $f=\left[\psi_{x}\left(\eta_{x}+\frac{1}{2} \psi_{x}^{2}\right)-\widetilde{\psi}_{x}\left(\widetilde{\eta}_{x}+\frac{1}{2} \widetilde{\psi}_{x}^{2}\right)\right]_{x}$ and $g=\frac{1}{2}\left(\psi_{x}^{2}-\widetilde{\psi}_{x}^{2}\right)_{x}$. Adding and subtracting the term $\widetilde{\psi}_{x}\left(\eta_{x}+\frac{1}{2} \psi_{x}^{2}\right)$ inside the norm $|f|$, one gets

$$
\begin{aligned}
|f| \leq & \left\|\psi_{x}-\widetilde{\psi}_{x}\right\|_{L^{\infty}(0, L)}\left|\eta_{x}+\frac{1}{2} \psi_{x}^{2}\right|+\left\|\widetilde{\psi}_{x}\right\|_{L^{\infty}(0, L)}\left|\eta_{x}-\widetilde{\eta}_{x}\right| \\
& +\frac{1}{2}\left\|\widetilde{\psi}_{x}\right\|_{L^{\infty}(0, L)}\left|\psi_{x}+\widetilde{\psi}_{x}\right|\left\|\psi_{x}-\widetilde{\psi}_{x}\right\|_{L^{\infty}(0, L)} .
\end{aligned}
$$


Using the embedding of $H^{1}(0, L)$ into $L^{\infty}(0, L)$, one has from (16) that

$$
|f| \leq C\left(\|U\|_{\mathcal{X}},\|\widetilde{U}\|_{\mathcal{X}}\right)\|U-\widetilde{U}\|_{\mathcal{X}} .
$$

Using once again the embedding of $H^{1}(0, L)$ into $L^{\infty}(0, L)$, one also sees that

$$
|g| \leq C\left(\|U\|_{\mathcal{X}},\|\widetilde{U}\|_{\mathcal{X}}\right)\|U-\widetilde{U}\|_{\mathcal{X}} .
$$

Combining (15), (17) and (18), it follows that $F(U)$ is locally Lipschitz continuous in $\mathcal{X}$.

Global existence in our case is a consequence of the total energy to be conservative, that is, $E_{k}^{\prime}(t)=0$ or $E_{k}(t)=E_{k}(0)$ for all $t \geq 0$. This concludes the result.

Remark 2 Theorem 1 guarantees the existence and uniqueness of finite-energy solutions for initial data $\left\{\phi_{0}, \phi_{1}, \psi_{0}, \psi_{1}, \eta_{0}, \eta_{1}\right\} \in \mathcal{X}$. In particular, we have considered

$$
\int_{0}^{L} \eta_{0}(x) d x=\int_{0}^{L} \eta_{1}(x) d x=0 .
$$

This condition is not necessary to obtain a unique finite-energy solution. Indeed, integrating equation $(3)_{3}$ with respect to $x$, we have

$$
\frac{d^{2}}{d t^{2}} \int_{0}^{L} \eta(x, t) d x=0
$$

which implies

$$
\int_{0}^{L} \eta(x, t) d x=\int_{0}^{L} \eta_{0}(x) d x+t \int_{0}^{L} \eta_{1}(x) d x .
$$

According to (20), the unique solution of the system is easily constructed without the condition (19). We set

$$
\mid \begin{aligned}
& \phi=\widetilde{\phi}, \quad \psi=\widetilde{\psi} \\
& \eta=\frac{1}{L} \int_{0}^{L} \eta_{0}(x) d x+\frac{t}{L} \int_{0}^{L} \eta_{1}(x) d x+\widetilde{\eta}
\end{aligned}
$$

where $\{\widetilde{\phi}, \widetilde{\psi}, \widetilde{\eta}\}$ is the unique solution provided by Theorem 1 associated to initial data $\left\{\phi_{0}, \phi_{1}, \psi_{0}, \psi_{1}, \widetilde{\eta}_{0}, \widetilde{\eta}_{1}\right\}$, with

$$
\widetilde{\eta}_{0}=\eta_{0}-\frac{1}{L} \int_{0}^{L} \eta_{0}(x) d x \quad \text { and } \quad \widetilde{\eta}_{1}=\eta_{1}-\frac{1}{L} \int_{0}^{L} \eta_{1}(x) d x,
$$

which clearly satisfy (19). Even though the zero average condition (19) is not necessary to show existence of solutions, it seems necessary to assure the existence of a solution defined in the whole interval $[0, \infty)$ (see $(21))$. This is why we require $(19)$, since we are also interested in studying the asymptotic behavior of solutions as $t \rightarrow \infty$ (see Section 4 ).

\section{Asymptotic Limit}

In this section, we study the asymptotic limit of the solutions for the nonlinear MindlinTimoshenko system (3), (6), (7) as $k \rightarrow \infty$. 
Theorem 2 Let $\left\{\phi^{k}, \psi^{k}, \eta^{k}\right\}$ be the unique solution of (3), (6), (7) with data $\left\{\phi_{0}, \phi_{1}, \psi_{0}, \psi_{1}, \eta_{0}, \eta_{1}\right\} \in \mathcal{X}$ satisfying

$$
\phi_{0}+\psi_{0 x}=0 \quad \text { in } \quad(0, L) .
$$

Then, letting $k \rightarrow \infty$, one gets

$$
\left\{\phi^{k}, \psi^{k}, \eta^{k}\right\} \rightarrow\left\{-\psi_{x}, \psi, \eta\right\} \text { weak }-* \text { in } L^{\infty}\left(0, T ;\left[H_{0}^{1}(0, L)\right]^{2} \times V\right),
$$

where $\{\psi, \eta\}$ solves the von Kármán system:

$$
\begin{cases}\rho h \psi_{t t}-\frac{\rho h^{3}}{12} \psi_{x x t t}+\psi_{x x x x}-\left[\psi_{x}\left(\eta_{x}+\frac{1}{2} \psi_{x}^{2}\right)\right]_{x}=0 & \text { in } \quad Q, \\ \rho h \eta_{t t}-\left(\eta_{x}+\frac{1}{2} \psi_{x}^{2}\right)_{x}=0 & \text { in } \quad Q \\ \psi(0, \cdot)=\psi(L, \cdot)=\psi_{x}(0, \cdot)=\psi_{x}(L, \cdot)=0 & \text { on } \quad(0, T), \\ \eta_{x}(0, \cdot)=\eta_{x}(L, \cdot)=0 & \text { on } \quad(0, T), \\ \psi(\cdot, 0)=\psi_{0}, \quad\left(\psi-\frac{h^{2}}{12} \psi_{x x}\right)_{t}(0)=\psi_{1}+\frac{h^{2}}{12} \phi_{1 x} & \text { in } \quad(0, L), \\ \eta(\cdot, 0)=\eta_{0}, \quad \eta_{t}(\cdot, 0)=\eta_{1} & \text { in } \quad(0, L) .\end{cases}
$$

Remark 3 Existence and uniqueness of weak solutions for the limit system (23) was proved in [12]. More precisely, when $\left\{\psi_{0}, \psi_{1}, \eta_{0}, \eta_{1}\right\} \in H_{0}^{2}(0, L) \times L^{2}(0, L) \times V \times H$, there exists a unique solution $\{\psi, \eta\}$ in the class

$$
\left\{\psi, \psi_{t}, \eta, \eta_{t}\right\} \in C^{0}\left([0, \infty) ; H_{0}^{2}(0, L) \times H_{0}^{1}(0, L) \times V \times H\right),
$$

satisfying the variational formulation of system (23)

$$
\begin{aligned}
& \rho h \frac{d}{d t}\left(\psi_{t}(t), w\right)+\frac{\rho h^{3}}{12} \frac{d}{d t}\left(\psi_{x}(t), w_{x}\right)+\rho h \frac{d}{d t}\left(\eta_{t}(t), y\right)+\left(\psi_{x x}(t), w_{x x}\right) \\
& +\left(\psi_{x}(t)\left(\eta_{x}(t)+\frac{1}{2}\left[\psi_{x}(t)\right]^{2}\right), w_{x}\right)+\left(\eta_{x}(t)+\frac{1}{2}\left[\psi_{x}(t)\right]^{2}, y_{x}\right)=0
\end{aligned}
$$

for all $\{w, y\} \in H_{0}^{2}(0, L) \times H^{1}(0, L)$ and the initial conditions $(23)_{5},(23)_{6}$. In $(24),(\cdot, \cdot)$ represents the inner product in $L^{2}(0, L)$. Furthermore, the energy $E(t)$ of system $(23)$

$$
\begin{gathered}
E(t)=\frac{1}{2}\left(\rho h\left|\psi_{t}(t)\right|^{2}+\rho h\left|\eta_{t}(t)\right|^{2}+\frac{\rho h^{3}}{12}\left|\psi_{x t}(t)\right|^{2}+\left|\psi_{x x}(t)\right|^{2}\right. \\
\left.+\left|\eta_{x}(t)+\frac{1}{2}\left[\psi_{x}(t)\right]^{2}\right|^{2}\right)
\end{gathered}
$$

is conservative, that is, $E(t)=E(0)$, for all $t \in[0, T]$.

Remark 4 Note that, in order to fully identify the initial data of the solutions of the limit system (23) and, more precisely, to determine the initial data of $\psi_{t}$, an elliptic equation has to be solved. Namely, the initial datum for the velocity $\psi_{t}$ in $(23)_{5}$ is determined by solving the elliptic equation

$$
\psi_{t}(\cdot, 0) \in H_{0}^{1}(0, L) ; \quad \rho h \psi_{t}(0)-\frac{\rho h^{3}}{12} \psi_{x x t}(0)=\rho h \psi_{1}+\frac{\rho h^{3}}{12} \phi_{1 x},
$$


as the proof of the theorem will show. More precisely, this elliptic equation can be written in the variational form

$$
-\frac{\rho h^{3}}{12}\left(\psi_{x t}(0), w_{x}\right)-\rho h\left(\psi_{t}(0), w\right)=\frac{\rho h^{3}}{12}\left(\phi_{1}, w_{x}\right)-\rho h\left(\psi_{1}, w\right), \forall w \in H_{0}^{1}(0, L),
$$

where the term $\phi_{1 x}$, which is an element of $H^{-1}(0, L)$, is not the derivative of $\phi_{1}$ in the sense of transposition, but rather the linear mapping which, when acting on any element $w$ of $H_{0}^{1}(0, L)$, yields the value $-\left(\phi_{1}, w_{x}\right)$. The same can be said about $\psi_{x x t}(0)$ which represents the element of $H^{-1}(0, L)$ yielding $-\left(\psi_{x t}(0), w_{x}\right)$.

Remark 5 According to Remark 2, one sees that condition (19) is not necessary to obtain the von Kármán system (23) as an asymptotic limit (as $k \rightarrow \infty$ ) of the perturbed MindlinTimoshenko system (3), (6), (7). Here, we assume again the average zero condition (19) only to assure the existence of solutions defined in the whole interval $[0, \infty)$ to be able to study its asymptotic behavior. This study will be carried out in the next section.

Proof of Theorem 2 For each $k>0$ fixed, let $\left\{\phi^{k}, \psi^{k}, \eta^{k}\right\}$ be the solution of system (3), (6), (7) with data $\left\{\phi_{0}, \phi_{1}, \psi_{0}, \psi_{1}, \eta_{0}, \eta_{1}\right\} \in \mathcal{X}$. We are interested in the asymptotic limit, as $k \rightarrow \infty$, of system $(3),(6),(7)$. Since the initial data $\left\{\phi_{0}, \phi_{1}, \psi_{0}, \psi_{1}, \eta_{0}, \eta_{1}\right\}$ satisfy the condition $(22)$, one has, due to the conservation of energy (10), that

$$
E_{k}(0) \leq C_{0}, \forall k>0 \text {. }
$$

Therefore, the sequences (in $k$ )

$$
\left(\phi^{k}\right),\left(\frac{1}{\sqrt{k}} \psi^{k}\right),\left(\eta^{k}\right)
$$

are bounded in $L^{\infty}\left(0, T ; H_{0}^{1}(0, L)\right), L^{\infty}\left(0, T ; H_{0}^{2}(0, L)\right)$, and $L^{\infty}(0, T ; V)$, respectively, and

$$
\left(\phi_{t}^{k}\right),\left(\psi_{t}^{k}\right),\left(\eta_{t}^{k}\right),\left(\sqrt{k}\left(\phi^{k}+\psi_{x}^{k}\right)\right),\left(\eta_{x}^{k}+\frac{1}{2}\left(\psi_{x}^{k}\right)^{2}\right)
$$

remain bounded in $L^{\infty}\left(0, T ; L^{2}(0, L)\right)$. Extracting subsequences, without changing notation, one gets

$$
\begin{gathered}
\frac{1}{\sqrt{k}} \psi^{k} \rightarrow \alpha \text { weak }-* \text { in } L^{\infty}\left(0, T ; H_{0}^{2}(0, L)\right), \\
\left\{\phi^{k}, \psi^{k}, \eta^{k}\right\} \rightarrow\{\phi, \psi, \eta\} \text { weak }-* \text { in } L^{\infty}\left(0, T ;\left[H_{0}^{1}(0, L)\right]^{2} \times V\right),
\end{gathered}
$$

with

$$
\phi+\psi_{x}=0
$$

Moreover,

$$
\left\{\phi_{t}^{k}, \psi_{t}^{k}, \eta_{t}^{k}\right\} \rightarrow\left\{\phi_{t}, \psi_{t}, \eta_{t}\right\} \text { weak }-* \text { in } L^{\infty}\left(0, T ;\left[L^{2}(0, L)\right]^{3}\right)
$$

and

$$
\eta_{x}^{k}+\frac{1}{2}\left(\psi_{x}^{k}\right)^{2} \rightarrow \xi \text { weak }-* \text { in } L^{\infty}\left(0, T ; L^{2}(0, L)\right)
$$

Now, using (29), we also get

$$
\frac{1}{k} \psi^{k}=\frac{1}{\sqrt{k}} \frac{1}{\sqrt{k}} \psi^{k} \rightarrow 0 \text { weak }-* \text { in } L^{\infty}\left(0, T ; H_{0}^{2}(0, L)\right)
$$


According to (26), we have, for $k \geq 1$, the following estimate:

$$
\frac{1}{\sqrt{k}}\left\|\phi^{k}(t)+\psi_{x}^{k}(t)\right\|_{H_{0}^{1}(0, L)} \leq\left|\phi_{x}^{k}(t)\right|+\frac{1}{\sqrt{k}}\left|\psi_{x x}^{k}(t)\right| \leq C .
$$

The character $C$ stands for a generic positive constant which may vary from line to line (unless otherwise stated). By interpolation, we can deduce that

$$
\begin{aligned}
& \left\|\phi^{k}(t)+\psi_{x}^{k}(t)\right\|_{H^{1 / 2}(0, L)} \\
\leq & C\left(k^{1 / 4}\left|\phi^{k}(t)+\psi_{x}^{k}(t)\right|^{1 / 2}\right)\left(k^{-1 / 4}\left\|\phi^{k}(t)+\psi_{x}^{k}(t)\right\|_{H_{0}^{1}(0, L)}^{1 / 2}\right) .
\end{aligned}
$$

Combining (26), (35), and (36), we obtain

$$
\left\|\psi_{x}^{k}(t)\right\|_{H^{1 / 2}(0, L)} \leq C .
$$

We also have by (26) that

$$
\left\|\psi^{k}(t)\right\|_{H^{1 / 2}(0, L)} \leq C\left\|\psi^{k}\right\|_{H^{1}(0, L)} \leq C\left(\sqrt{k}\left|\phi^{k}+\psi_{x}^{k}\right|+\left|\phi^{k}\right|\right) \leq C .
$$

Since the norms

$$
\|u\|_{H^{3 / 2}(0, L)} \quad \text { and } \quad\left(\|u\|_{H^{1 / 2}(0, L)}^{2}+\left\|u_{x}\right\|_{H^{1 / 2}(0, L)}^{2}\right)^{1 / 2}
$$

are equivalent (see [14, Theorem 9.7]), we get by $(37)$ and (38) that the sequence $\left(\psi^{k}\right)$ in $L^{\infty}\left(0, T ; H_{0}^{3 / 2}(0, L)\right) \cap W^{1, \infty}\left(0, T ; L^{2}(0, L)\right)$ is uniformly bounded with respect to $k$. In this way, due to the Aubin-Lions compactness theorem (see [15, Corollary 4]), we obtain

$$
\psi^{k} \rightarrow \psi \text { strongly in } L^{\infty}\left(0, T ; H^{\frac{3}{2}-\delta}(0, L)\right),
$$

for any $\delta>0$ which, by Sobolev's embeddings, implies the strong convergence in $L^{\infty}\left(0, T ; L^{p}(0, L)\right)$, for all $1 \leq p<\infty$.

Combining (30) and (39), it follows that $\xi=\eta_{x}+\psi_{x}^{2} / 2$, and

$$
\psi_{x}^{k}\left[\eta_{x}^{k}+\frac{1}{2}\left(\psi_{x}^{k}\right)^{2}\right] \rightarrow \psi_{x}\left[\eta_{x}+\frac{1}{2} \psi_{x}^{2}\right] \text { weakly in } L^{2}(Q) .
$$

For $\{z, w, y\} \in H_{0}^{1}(0, L) \times H_{0}^{2}(0, L) \times H^{1}(0, L)$ satisfying

$$
z+w_{x}=0,
$$

the variational formulation of (3), (6), (7) is simply

$$
\begin{aligned}
& \frac{\rho h^{3}}{12} \frac{d}{d t}\left(\phi_{t}^{k}(t), z\right)+\rho h \frac{d}{d t}\left(\psi_{t}^{k}(t), w\right)+\rho h \frac{d}{d t}\left(\eta_{t}^{k}(t), y\right)+\left(\phi_{x}^{k}(t), z_{x}\right) \\
& +\left(\psi_{x}^{k}(t)\left(\eta_{x}^{k}(t)+\frac{1}{2}\left[\psi_{x}^{k}(t)\right]^{2}\right), w_{x}\right)+\left(\eta_{x}^{k}(t)+\frac{1}{2}\left[\psi_{x}^{k}(t)\right]^{2}, y_{x}\right) \\
& +\frac{1}{k}\left(\psi_{x x}^{k}(t), w_{x x}\right)=0 .
\end{aligned}
$$

Using convergences (30), (32) - (34) and (40) in equation (42), and applying identities (31) and (41), one obtains the weak formulation of the system (23) given in (24). To finish the proof, 
it remains to identify the initial data of the limit system. In view of the convergences (30), (32), and classical compactness arguments, one has $\left\{\psi^{k}, \eta^{k}\right\} \rightarrow\{\psi, \eta\}$ in $C^{0}\left([0, T] ;\left[L^{2}(0, L)\right]^{2}\right)$. Then, $\left\{\psi^{k}(\cdot, 0), \eta^{k}(\cdot, 0)\right\} \rightarrow\{\psi(\cdot, 0), \eta(\cdot, 0)\}$ in $\left[L^{2}(0, L)\right]^{2}$, which combined with $(7)_{1}$, guarantees that $\{\psi(\cdot, 0), \eta(\cdot, 0)\}=\left\{\psi_{0}, \eta_{0}\right\}$. In order to identify $\left\{\psi_{t}(\cdot, 0), \eta_{t}(\cdot, 0)\right\}$, multiply both sides of equation (42) by the function $\theta_{\delta} \in H^{1}(0, T)$ defined by

$$
\theta_{\delta}(t)=\left\{\begin{array}{cl}
-\frac{t}{\delta}+1, & \text { if } \quad t \in[0, \delta] \\
0, & \text { if } \quad t \in(\delta, T]
\end{array}\right.
$$

and integrate by parts to obtain

$$
\begin{aligned}
& -\frac{\rho h^{3}}{12}\left(\phi_{1}, z\right)+\frac{\rho h^{3}}{12 \delta} \int_{0}^{\delta}\left(\phi_{t}^{k}(t), z\right) d t-\rho h\left(\psi_{1}, w\right)+\frac{\rho h}{\delta} \int_{0}^{\delta}\left(\psi_{t}^{k}(t), w\right) d t \\
& -\rho h\left(\eta_{1}, y\right)+\rho h \int_{0}^{\delta}\left(\eta_{t}^{k}(t), y\right) d t+\int_{0}^{\delta}\left(\phi_{x}^{k}(t), z_{x}\right) \theta_{\delta}(t) d t+\frac{1}{k} \int_{0}^{\delta}\left(\psi_{x x}^{k}(t), w_{x x}\right) \theta_{\delta}(t) d t \\
& +\int_{0}^{\delta}\left(\psi_{x}^{k}(t)\left(\eta_{x}^{k}(t)+\frac{1}{2}\left[\psi_{x}^{k}(t)\right]^{2}\right), w_{x}\right) \theta_{\delta}(t) d t+\int_{0}^{\delta}\left(\eta_{x}^{k}(t)+\frac{1}{2}\left[\psi_{x}^{k}(t)\right]^{2}, y_{x}\right) \theta_{\delta}(t) d t=0 .
\end{aligned}
$$

Passing to the limit as $k \rightarrow \infty$ in the last equation, and using (30), (32)-(34) and (40), one obtains

$$
\begin{aligned}
& \frac{\rho h^{3}}{12}\left(\phi_{1}, w_{x}\right)-\rho h\left(\psi_{1}, w\right)-\rho h\left(\eta_{1}, y\right)+\frac{\rho h^{3}}{12 \delta} \int_{0}^{\delta}\left(\psi_{x t}(t), w_{x}\right) d t \\
& +\frac{\rho h}{\delta} \int_{0}^{\delta}\left(\psi_{t}(t), w\right) d t+\rho h \int_{0}^{\delta}\left(\eta_{t}(t), y\right) d t+\int_{0}^{\delta}\left(\psi_{x x}(t), w_{x x}\right) \theta_{\delta}(t) d t \\
& +\int_{0}^{\delta}\left(\psi_{x}(t)\left(\eta_{x}(t)+\frac{1}{2}\left[\psi_{x}(t)\right]^{2}\right), w_{x}\right) \theta_{\delta}(t) d t+\int_{0}^{\delta}\left(\eta_{x}(t)+\frac{1}{2}\left[\psi_{x}(y)\right]^{2}, y_{x}\right) \theta_{\delta}(t) d t=0
\end{aligned}
$$

On the other hand, multiplying Equation (24) by $\theta_{\delta}$ and integrating in time we get the identity

$$
\begin{aligned}
& -\frac{\rho h^{3}}{12}\left(\psi_{x x t}(0), w\right)+\rho h\left(\psi_{t}(0), w\right)+\rho h\left(\eta_{t}(0), y\right) \\
= & \frac{\rho h^{3}}{12}\left(\phi_{1 x}, w\right)+\rho h\left(\psi_{1}, w\right)+\rho h\left(\eta_{1}, y\right), \quad \forall\{w, y\} \in H_{0}^{2}(0, L) \times H^{1}(0, L) .
\end{aligned}
$$

In this way, we conclude that $\left(\psi-\frac{h^{2}}{12} \psi_{x x}\right)_{t}(0)=\psi_{1}+\frac{h^{2}}{12} \phi_{1 x}$, and $\eta_{t}(0)=\eta_{1}$.

\section{Uniform Stabilization}

The aim of this section is to obtain the exponential decay for the energy (25) associated to the solution of the von Kármán system

$$
\begin{cases}\rho h \psi_{t t}-\frac{\rho h^{3}}{12} \psi_{x x t t}+\psi_{x x x x}-\left[\psi_{x}\left(\eta_{x}+\frac{1}{2} \psi_{x}^{2}\right)\right]_{x}+\psi_{t}-\psi_{x x t}=0 & \text { in } \quad Q, \\ \rho h \eta_{t t}-\left(\eta_{x}+\frac{1}{2} \psi_{x}^{2}\right)_{x}+\eta_{t}=0 & \text { in } \quad Q, \\ \psi(0, \cdot)=\psi(L, \cdot)=\psi_{x}(0, \cdot)=\psi_{x}(L, \cdot)=0 & \text { on }(0, T), \\ \eta_{x}(0, \cdot)=\eta_{x}(L, \cdot)=0 & \text { on }(0, T), \\ \left\{\psi(\cdot, 0), \psi_{t}(\cdot, 0), \eta(\cdot, 0), \eta_{t}(\cdot, 0)\right\}=\left\{\psi_{0}, \psi_{1}, \eta_{0}, \eta_{1}\right\} & \text { in }(0, L),\end{cases}
$$


as a limit (as $k \rightarrow \infty$ ) of the uniform stabilization of the perturbed Mindlin-Timoshenko system

$$
\left\{\begin{array}{lr}
\frac{\rho h^{3}}{12} \phi_{t t}-\phi_{x x}+k\left(\phi+\psi_{x}\right)+\phi_{t}=0 & \text { in } Q, \\
\rho h \psi_{t t}-k\left(\phi+\psi_{x}\right)_{x}-\left[\psi_{x}\left(\eta_{x}+\frac{1}{2} \psi_{x}^{2}\right)\right]_{x}+\frac{1}{k} \psi_{x x x x}+\psi_{t}=0 & \text { in } Q, \\
\rho h \eta_{t t}-\left(\eta_{x}+\frac{1}{2} \psi_{x}^{2}\right)_{x}+\eta_{t}=0 & \text { in } Q, \\
\phi(0, \cdot)=\phi(L, \cdot)=0 & \text { on }(0, T), \\
\psi(0, \cdot)=\psi(L, \cdot)=0 & \text { on }(0, T), \\
\eta_{x}(0, \cdot)=\eta_{x}(L, \cdot)=0 & \text { on }(0, T), \\
\{\phi(\cdot, 0), \psi(\cdot, 0), \eta(\cdot, 0)\}=\left\{\phi_{0}, \psi_{0}, \eta_{0}\right\} & \text { in }(0, L), \\
\left\{\phi_{t}(\cdot, 0), \psi_{t}(\cdot, 0), \eta_{t}(\cdot, 0)\right\}=\left\{\phi_{1}, \psi_{1}, \eta_{1}\right\} & \text { in }(0, L) .
\end{array}\right.
$$

Analogously to the proof of Theorem 2, the system (45) can be obtained as a limit, when $k \rightarrow \infty$, of system (46). Notice that its energy given by (9) obeys the energy dissipation law

$$
E_{k}^{\prime}(t)=-\left(\left|\phi_{t}(t)\right|^{2}+\left|\psi_{t}(t)\right|^{2}+\left|\eta_{t}(t)\right|^{2}\right),
$$

that is, $E_{k}(t)$ is a non increasing function. We will show that this energy decays exponentially (as $t \rightarrow \infty$ ) uniformly with respect to $k$. More precisely, the following result holds.

Theorem 3 Let $\{\phi, \psi, \eta\}$ be the global solution of system (46) for data $\left\{\phi_{0}, \phi_{1}, \psi_{0}, \psi_{1}, \eta_{0}, \eta_{1}\right\}$ in $\mathcal{X}$ satisfying (22). Then there exists a constant $\omega>0$ such that

$$
E_{k}(t) \leq 4 E_{k}(0) \mathrm{e}^{-\frac{\omega}{2} t}, \quad \forall t \geq 0
$$

Remark 6 As a consequence of inequality (48), letting $k \rightarrow \infty$ one recovers the exponential decay of the energy $E(t)$, associated to system (45), and given by (25). This is in agreement with the results obtained in [10].

Remark 7 Note that the zero average condition (19) is necessary to prove Theorem 3, since one needs to assure all time existence of solutions. It is also important to note that the zero average property is preserved for the damped system (46), that is, its solutions have zero average as well. Indeed, integrating equation $(46)_{3}$ with respect to $x$, we get

$$
\frac{d^{2}}{d t^{2}} \int_{0}^{L} \eta(x, t) d x+\frac{d}{d t} \int_{0}^{L} \eta(x, t) d x=0
$$

which implies

$$
\int_{0}^{L} \eta(x, t) d x=\int_{0}^{L} \eta_{0}(x) d x+\int_{0}^{L} \eta_{1}(x) d x-\left(\int_{0}^{L} \eta_{1}(x) d x\right) \mathrm{e}^{-t}=0 .
$$

Proof of Theorem 3 For each $k \geq 1$ fixed, let $\left\{\phi^{k}, \psi^{k}, \eta^{k}\right\}$ be the solution of system (3), (6), (7) with data $\left\{\phi_{0}, \phi_{1}, \psi_{0}, \psi_{1}, \eta_{0}, \eta_{1}\right\} \in \mathcal{X}$. From now on, in this proof, we will omit the index $k$ of the solution to simplify the notation. For an arbitrary $\lambda>0$, define the perturbed energy

$$
G_{\lambda}(t)=E_{k}(t)+\lambda F(t)
$$

where $F$ is the functional

$$
F(t)=\theta\left(\frac{\rho h^{3}}{12} \phi_{t}(t), \phi(t)\right)+\theta\left(\rho h \psi_{t}(t), \psi(t)\right)+2 \theta\left(\rho h \eta_{t}(t), \eta(t)\right),
$$

for $\theta>0$ a constant to be chosen later on. Let us bound each term on the right hand side of identity (50) by an expression involving the energy (9). 
- Analysis of $\theta\left(\frac{\rho h^{3}}{12} \phi_{t}(t), \phi(t)\right)$.

Using Poincaré inequality, one gets

$$
\theta\left(\frac{\rho h^{3}}{12} \phi_{t}(t), \phi(t)\right) \leq \frac{\theta}{2} \sqrt{\frac{\rho h^{3} L}{12}}\left(\frac{\rho h^{3}}{12}\left|\phi_{t}(t)\right|^{2}+\left|\phi_{x}(t)\right|^{2}\right) \leq \theta \sqrt{\frac{\rho h^{3} L}{12}} E_{k}(t) .
$$

- Analysis of $\theta\left(\rho h \psi_{t}(t), \psi(t)\right)$.

Using Poincaré inequality as well, one gets

$$
\begin{aligned}
\theta\left(\rho h \psi_{t}(t), \psi(t)\right) & \leq \theta \rho h \sqrt{L}\left|\psi_{t}(t)\right|\left(\left|\phi(t)+\psi_{x}(t)\right|+\sqrt{L}\left|\phi_{x}(t)\right|\right) \\
& \leq \theta\left(\sqrt{\rho h L}+\sqrt{\rho h L^{2}}\right) E_{k}(t) .
\end{aligned}
$$

- Analysis of $2 \theta\left(\rho h \eta_{t}(t), \eta(t)\right)$.

One has

$$
2 \theta\left(\rho h \eta_{t}(t), \eta(t)\right) \leq 2 \theta \rho h\left|\eta_{t}(t)\right||\eta(t)| .
$$

Notice that the initial data satisfy (22). Thus, one can guarantee, due to (47), that the sequence $\left(\phi^{k}\right)$ is bounded in $L^{\infty}\left(0, T ; H_{0}^{1}(0, L)\right)$, and that $\left(\phi^{k}+\psi_{x}^{k}\right),\left(\eta_{x}^{k}+\frac{1}{2}\left(\psi_{x}^{k}\right)^{2}\right)$ are bounded in $L^{\infty}\left(0, T ; L^{2}(0, L)\right)$. Consequently, the sequence $\left(\psi_{x}^{k}\right)$ is bounded in $L^{\infty}\left(0, T ; L^{2}(0, L)\right)$. So, the sequence $\left(\left(\psi_{x}^{k}\right)^{2}\right)$ remains bounded in $L^{\infty}\left(0, T ; L^{1}(0, L)\right)$. One also has that $\left(\eta_{x}^{k}\right)$ is bounded in $L^{\infty}\left(0, T ; L^{1}(0, L)\right)$.

Since $\eta(t) \in V$ (Remark 7), one uses the Poincaré-Wirtinger inequality (see [16]) and gets

$$
|\eta(t)| \leq \sqrt{L}\left\|\eta_{x}(t)\right\|_{L^{1}(0, L)} \leq L\left|\eta_{x}(t)+\frac{1}{2}\left[\psi_{x}(t)\right]^{2}\right|+\frac{\sqrt{L}}{2}\left\|\left[\psi_{x}(t)\right]^{2}\right\|_{L^{1}(0, L)} .
$$

On the other hand,

$$
\begin{aligned}
& \frac{\sqrt{L}}{2}\left\|\left[\psi_{x}(t)\right]^{2}\right\|_{L^{1}(0, L)} \\
\leq & \frac{\sqrt{L}}{2}\left|\psi_{x}(t)\right|\left|\psi_{x}(t)\right| \\
\leq & \frac{\sqrt{L}}{2}\left(\left|\phi(t)+\psi_{x}(t)\right|+\sqrt{L}\left|\phi_{x}(t)\right|\right)\left(\left|\phi(t)+\psi_{x}(t)\right|+\sqrt{L}\left|\phi_{x}(t)\right|\right) \\
\leq & \frac{(1+L) \sqrt{L}}{\sqrt{2}} \sqrt{E_{k}(t)}\left(\left|\phi(t)+\psi_{x}(t)\right|+\sqrt{L}\left|\phi_{x}(t)\right|\right) \\
\leq & \frac{(1+L) \sqrt{L C_{0}}}{\sqrt{2}}\left(\left|\phi(t)+\psi_{x}(t)\right|+\sqrt{L}\left|\phi_{x}(t)\right|\right),
\end{aligned}
$$

where $C_{0}$ is given in (26). Therefore, it follows by (53) - (55) that

$$
\begin{aligned}
& 2 \theta\left(\rho h \eta_{t}(t), \eta(t)\right) \\
\leq & \theta(1+L) \sqrt{2 L^{2} C_{0}} \rho h\left|\eta_{t}(t)\right|\left|\phi_{x}(t)\right|+2 \theta L \rho h\left|\eta_{t}(t)\right|\left|\eta_{x}(t)+\frac{1}{2}\left[\psi_{x}(t)\right]^{2}\right| \\
& +\theta(1+L) \sqrt{2 L C_{0}} \rho h\left|\eta_{t}(t)\right|\left|\phi(t)+\psi_{x}(t)\right| \\
\leq & \theta\left[(1+L) \sqrt{2 \rho h L^{2} C_{0}}+2 \sqrt{\rho h L^{2}}+(1+L) \sqrt{2 \rho h L C_{0}}\right] E_{k}(t) .
\end{aligned}
$$


According to the bounds (51), (52), and (56), one has

$$
|F(t)| \leq C_{1} E_{k}(t),
$$

where $C_{1}=\theta \sqrt{\rho h L}\left[1+3 \sqrt{L}+\sqrt{1 / 12} h+(1+L)(1+\sqrt{L}) \sqrt{2 C_{0}}\right]$. Now, using (49) and (57), one has

$$
\left|G_{\lambda}(t)-E_{k}(t)\right| \leq \lambda|F(t)| \leq \lambda C_{1} E_{k}(t),
$$

which is equivalent to

$$
\left(1-\lambda C_{1}\right) E_{k}(t) \leq G_{\lambda}(t) \leq\left(1+\lambda C_{1}\right) E_{k}(t)
$$

Taking $0<\lambda \leq 1 / 2 C_{1}$, one gets

$$
\frac{E_{k}(t)}{2} \leq G_{\lambda}(t) \leq 2 E_{k}(t)
$$

Differentiating the functional $F$ and using the equations in (46), one obtains

$$
\begin{aligned}
F^{\prime}(t)= & -\theta\left|\phi_{x}(t)\right|^{2}-\theta k\left|\phi(t)+\psi_{x}(t)\right|^{2}-2 \theta\left|\eta_{x}+\frac{1}{2}\left[\psi_{x}(t)\right]^{2}\right|^{2} \\
& +\frac{\theta \rho h^{3}}{12}\left|\phi_{t}(t)\right|^{2}+\theta \rho h\left|\psi_{t}(t)\right|^{2}+2 \theta \rho h\left|\eta_{t}(t)\right|^{2} \\
& -\theta\left(\phi_{t}(t), \phi(t)\right)+\theta\left(\psi_{t}(t), \psi(t)\right)-2 \theta\left(\eta_{t}(t), \eta(t)\right) .
\end{aligned}
$$

We bound each term that appears on the right hand side of the identity (59) separately.

- Analysis of $-\theta\left(\phi_{t}(t), \phi(t)\right)$.

$$
-\theta\left(\phi_{t}(t), \phi(t)\right) \leq C_{2}\left|\phi_{t}(t)\right|^{2}+\frac{\xi}{2}\left|\phi_{x}(t)\right|^{2} \leq C_{2}\left|\phi_{t}(t)\right|^{2}+\xi E_{k}(t),
$$

where $C_{2}=\theta^{2} L / 2 \xi$ and $\xi>0$ is a real number to be appropriately chosen.

- Analysis of $\theta\left(\psi_{t}(t), \psi(t)\right)$.

$$
\begin{aligned}
\theta\left(\psi_{t}(t), \psi(t)\right) & \leq C_{3}\left|\psi_{t}(t)\right|^{2}+\frac{\xi}{2}\left|\phi_{x}(t)\right|^{2}+\frac{\xi}{2} k\left|\phi(t)+\psi_{x}(t)\right|^{2} \\
& \leq C_{3}\left|\psi_{t}(t)\right|^{2}+\xi E_{k}(t)
\end{aligned}
$$

where $C_{3}=\theta^{2} L(1+L) / 2 \xi$.

- Analysis of $-2 \theta\left(\eta_{t}(t), \eta(t)\right)$.

It follows by inequalities (54) and (55) that

$$
\begin{aligned}
& -2 \theta\left(\eta_{t}(t), \eta(t)\right) \\
\leq & 2 \theta\left|\eta_{t}(t)\right||\eta(t)| \\
\leq & \theta(1+L) \sqrt{2 L^{2} C_{0}}\left|\eta_{t}(t)\right|\left|\phi_{x}(t)\right|+2 \theta L\left|\eta_{t}(t)\right|\left|\eta_{x}(t)+\frac{1}{2}\left[\psi_{x}(t)\right]^{2}\right| \\
& +\theta(1+L) \sqrt{2 L C_{0}}\left|\eta_{t}(t)\right|\left|\phi(t)+\psi_{x}(t)\right| \leq C_{4}\left|\eta_{t}(t)\right|^{2}+\xi E_{k}(t),
\end{aligned}
$$

where $C_{4}=\theta^{2} L\left[2 L+(1+L)^{3} C_{0}\right] / \xi$. 
Using bounds (60), (61), and (62), one obtains, from (59),

$$
\begin{aligned}
F^{\prime}(t) \leq & -\theta\left|\phi_{x}(t)\right|^{2}-\theta k\left|\phi(t)+\psi_{x}(t)\right|^{2}-2 \theta\left|\eta_{x}+\frac{1}{2}\left(\psi_{x}\right)^{2}\right|^{2}+\left(\frac{\theta \rho h^{3}}{12}+C_{2}\right)\left|\phi_{t}(t)\right|^{2} \\
& +\left(\theta \rho h+C_{3}\right)\left|\psi_{t}(t)\right|^{2}+\left(2 \theta \rho h+C_{4}\right)\left|\eta_{t}(t)\right|^{2}+3 \xi E_{k}(t) .
\end{aligned}
$$

Therefore,

$$
F^{\prime}(t) \leq-(\theta-3 \xi) E_{k}(t)+C_{5}\left|\phi_{t}(t)\right|^{2}+C_{6}\left|\psi_{t}(t)\right|^{2}+C_{7}\left|\eta_{t}(t)\right|^{2}
$$

where $C_{5}=\left(\theta \rho h^{3} / 12\right)+C_{2}, C_{6}=2 \theta \rho h+C_{3}$, and $C_{7}=2 \theta \rho h+C_{4}$.

Considering the derivative of the expression (49), and observing (47) and (63), one has

$$
G_{\lambda}^{\prime}(t) \leq-\lambda(\theta-3 \xi) E_{k}(t)-\left(1-\lambda C_{5}\right)\left|\phi_{t}(t)\right|^{2}-\left(1-\lambda C_{6}\right)\left|\psi_{t}(t)\right|^{2}-\left(1-\lambda C_{7}\right)\left|\eta_{t}(t)\right|^{2} .
$$

Taking $\lambda=\min \left\{1 / 2 C_{1}, 1 / C_{5}, 1 / C_{6}, 1 / C_{7}\right\}$ and $\xi<\theta / 3$, one obtains, according to (58),

$$
G_{\lambda}^{\prime}(t) \leq-\frac{\omega}{2} G_{\lambda}(t), \quad \forall t \geq 0
$$

where $\omega=\lambda(\theta-3 \xi)$. In this way,

$$
G_{\lambda}(t) \leq G_{\lambda}(0) \mathrm{e}^{-\frac{\omega}{2} t}, \quad \forall t \geq 0
$$

Combining (58) and (64), one gets (48). This finishes the proof.

For the original dissipative Mindlin-Timoshenko system

$$
\begin{cases}\frac{\rho h^{3}}{12} \phi_{t t}-\phi_{x x}+k\left(\phi+\psi_{x}\right)+\phi_{t}=0 & \text { in } \quad Q \\ \rho h \psi_{t t}-k\left(\phi+\psi_{x}\right)_{x}-\left[\psi_{x}\left(\eta_{x}+\frac{1}{2} \psi_{x}^{2}\right)\right]_{x}+\psi_{t}=0 & \text { in } \quad Q \\ \rho h \eta_{t t}-\left(\eta_{x}+\frac{1}{2} \psi_{x}^{2}\right)_{x}+\eta_{t}=0 & \text { in } \quad Q \\ \phi(0, \cdot)=\phi(L, \cdot)=0 & \text { on } \quad(0, T) \\ \psi(0, \cdot)=\psi(L, \cdot)=0 & \text { on } \quad(0, T) \\ \eta_{x}(0, \cdot)=\eta_{x}(L, \cdot)=0 & \text { on } \quad(0, T) \\ \{\phi(\cdot, 0), \psi(\cdot, 0), \eta(\cdot, 0)\}=\left\{\phi_{0}, \psi_{0}, \eta_{0}\right\} & \text { in } \quad(0, L) \\ \left\{\phi_{t}(\cdot, 0), \psi_{t}(\cdot, 0), \eta_{t}(\cdot, 0)\right\}=\left\{\phi_{1}, \psi_{1}, \eta_{1}\right\} & \text { in } \quad(0, L),\end{cases}
$$

one can proceed analogously to the proof of Theorem 3 to get the following

Theorem 4 Let $\{\phi, \psi, \eta\}$ be the global solution of system (65) for data $\left\{\phi_{0}, \phi_{1}, \psi_{0}, \psi_{1}, \eta_{0}, \eta_{1}\right\}$ in $\mathcal{X}$ satisfying $(22)$. Then there exists a constant $\widehat{\omega}>0$ such that the energy

$$
\begin{gathered}
\mathcal{E}_{k}(t)=\frac{1}{2}\left(\frac{\rho h^{3}}{12}\left|\phi_{t}(t)\right|^{2}+\rho h\left|\psi_{t}(t)\right|^{2}+\rho h\left|\eta_{t}(t)\right|^{2}+\left|\phi_{x}(t)\right|^{2}\right. \\
\left.+k\left|\phi(t)+\psi_{x}(t)\right|^{2}+\left|\eta_{x}(t)+\frac{1}{2}\left[\psi_{x}(t)\right]^{2}\right|^{2}\right)
\end{gathered}
$$


satisfies

$$
\mathcal{E}_{k}(t) \leq 4 \mathcal{E}_{k}(0) \mathrm{e}^{-\widehat{\widehat{\omega}} t}, \quad \forall t \geq 0
$$

Remark 8 Even though Theorem 4 provides a uniform (in $k$ ) stabilization result, passing to the limit in this system as $k \rightarrow \infty$ is an open problem, because of the lack of compactness of the quadratic term on $\psi_{x}$.

\section{Further Comments and Open Problems}

In this section we briefly discuss some possible extensions of our results and also indicate open problems on the subject.

1) The methods of this paper work in the context of model (3) in which a fourth order dispersive term has been added to ensure the compactness of the families of solutions as the singular parameter $k$ tends to infinity. It would be interesting to analyze whether the same results hold without that regularizing term. The difficulty, of course, consists in getting enough compactness to pass to the limit in the nonlinear term.

2) It would be interesting to analyze whether the results of this paper hold for different boundary conditions.

3) In the proof of Theorems 2 and 3, we have considered the case when the initial data $\phi_{0}, \phi_{1}, \psi_{0}, \psi_{1}, \eta_{0}$ and $\eta_{1}$ are fixed. The same results hold if we consider the case where they do depend on $k$, provided we assume that $\phi_{0}^{k}, \phi_{1}^{k}, \psi_{0}^{k}, \psi_{1}^{k}, \eta_{0}^{k}$ and $\eta_{1}^{k}$ are such that the initial energy $E_{k}(0)$ remains bounded and $\left\{\phi_{0}^{k}, \phi_{1}^{k}, \psi_{0}^{k}, \psi_{1}^{k}, \eta_{0}^{k}, \eta_{1}^{k}\right\}$ converges weakly to $\left\{\phi_{0}, \phi_{1}, \psi_{0}, \psi_{1}, \eta_{0}, \eta_{1}\right\}$ in the corresponding spaces.

4) One could address the problem of uniform stabilization with damping terms that are only active on the rotation angle and longitudinal displacement equations in the Mindlin-Timoshenko system, that is, systems (46) and (65) without adding the dissipative term $\psi_{t}$. This is a natural choice, since, in the limit, the dissipative term $\phi_{t}$ generates the damping $-\psi_{x x t}$ which acts in the vertical displacement equation of the von Kármán system and suffices to guarantee the exponential decay of the limit system. One could expect the energy of the approximating system to decay uniformly, with respect to the parameter $k$, as $t \rightarrow \infty$.

\section{References}

[1] J. F. Doyle, Wave Propagation in Structures, Springer-Verlag, New York, 1997.

[2] J. E. Lagnese, Boundary Stabilization of Thin Plates, SIAM, 1989.

[3] J. E. Lagnese and J. L. Lions, Modelling Analysis and Control of Thin Plates, RMA 6, Masson, Paris, 1988.

[4] I. Chueshov and I. Lasiecka, Global attractors for Mindlin-Timoshenko plates and for their Kirchhoff limits, Milan J. Math., 2006, 74: 117-138.

[5] A. Favini, M. A. Horn, I. Lasiecka, and D. Tartaru, Global existence, uniqueness and regularity of solutions to a von Kármán system with nonlinear boundary dissipation, Diff. Integ. Eqns, 1996, 9: $267-294$.

[6] J. E. Lagnese and G. Leugering, Uniform stabilization of a nonlinear beam by nonlinear boundary feedback, J. Diff. Eqns, 1991, 91: 355-388.

[7] D. L. Russell, Controllability and stabilizability theory for linear partial differential equations. Recent progress and open questions, SIAM Rev., 1978, 20: 639-739.

[8] J. L. Lions, Exact controllability, stabilizability and perturbations for distributed systems, SIAM Rev., 1988, 30: 1-68. 
[9] F. D. Araruna and E. Zuazua, Controllability of the Kirchhoff system for beams as a limit of the Mindlin-Timoshenko system, SIAM J. Cont. Optim., 2008, 47(4): 1909-1938.

[10] A. Pazoto, G. Perla Menzala, and E. Zuazua, Stabilization of Berger-Timoshenko's equation as limit of the uniform stabilization of the von Kárman system of beams and plates, Math. Model. Numer. Anal., 2002, 36(4): 657-691.

[11] G. Perla Menzala and E. Zuazua, The beam equation as a limit of 1-D nonlinear von Kármán model, Appl. Math. Lett., 1999, 12: 47-52.

[12] G. Perla Menzala and E. Zuazua, Timoshenko's beam equation as limit of a nonlinear onedimensional von Kármán system, Proceedings of the Royal Society of Edinburgh, 2000, 130A: 855-875.

[13] G. Perla Menzala and E. Zuazua, Timoshenko's plate equation as a singular limit of the dynamical von Kármán system, J. Math. Pures Appl., 2000, 79(1): 73-94.

[14] J. L. Lions and E. Magenes, Non-Homogeneous Boundary Value Problems and Applications, SpringerVerlag, New York, vol. 1, 1972.

[15] J. Simon, Compact sets in the space $L^{p}(0, T ; B)$, Ann. Mat. Pura Appl., 1987, CXLVI(4): 65-96.

[16] H. Brezis, Analyse Fonctionnelle: Theórie et Applications, Dunod, Paris, 1999.

[17] G. Perla Menzala and E. Zuazua, Explicity exponential decay rates for solutions of von Kármán system of thermoelastic plates, C. R. Acad. Sci. Paris, 1997, 324: 49-54. 